\title{
WELFake: Word Embedding over Linguistic Features for Fake News Detection
}

\author{
Pawan Kumar Verma Member, IEEE, Prateek Agrawal, Ivone Amorim and Radu Prodan Member, IEEE
}

\begin{abstract}
Social media is a popular medium for dissemination of real-time news all over the world. Easy and quick information proliferation is one of the reasons for its popularity. An extensive number of users with different age groups, gender and societal beliefs are engaged in social media websites. Despite these favorable aspects, a significant disadvantage comes in the form of fake news, as people usually read and share information without caring about its genuineness. Therefore, it is imperative to research methods for the authentication of news. To address this issue, this paper proposes a two phase benchmark model named WELFake based on word embedding (WE) over linguistic features for fake news detection using machine learning classification. The first phase pre-processes the dataset and validates the veracity of news content by using linguistic features. The second phase merges the linguistic feature sets with WE and applies voting classification. To validate its approach, this paper also carefully designs a novel WELFake dataset with approximately 72,000 articles, which incorporates different datasets to generate an unbiased classification output. Experimental results show that the WELFake model categorises the news in real and fake with a $96.73 \%$ which improves the overall accuracy by $1.31 \%$ compared to BERT and $4.25 \%$ compared to CNN models. Our frequencybased and focused analyzing writing patterns model outperforms predictive-based related works implemented using the Word2vec WE method by up to $1.73 \%$.
\end{abstract}

Index Terms-Fake news; text classification; machine learning; word embedding; linguistic feature; voting classifier; $\mathrm{CNN}$; BERT.

\section{INTRODUCTION}

$\mathbf{N}$ OWADAYS people around the world are getting much involved on online social networks regardless of age, community or sex [1]. Communicating using social networks is simple, fast and attractive to share and transfer information. Currently, social network sites like Facebook trailed by Twitter are the market pioneers, facilitating over 1.3 billion clients with a dynamic monthly variation of 300 million users in average [2]. Their collaborations generate Terabytes of information every second [3], [4]. Online social networks are attractive because of the simple and convenient way to access and circulate information with other people. However, fast

Pawan Kumar Verma is with the Department of Computer Science and Engineering, GLA University, and also with the Lovely Professional University, India. Email: pawankumar.verma@gla.ac.in

Prateek Agrawal is with the School of Computer Science and Engineering, Lovely Professional University, India, and also with the Institute of Information Technology, University of Klagenfurt, Austria. Email: prateek.agrawal@1pu.co.in

Ivone Amorim is with MOG Technologies, and also with the CMUP mathematical research center, University of Porto, Portugal. Email: ivone.amorim@mog-technologies.com

Radu Prodan is with the Institute of Information Technology, University of Klagenfurt, Austria. Email: radu@itec.aau.at scattering of data at a high rate with minimal effort enables the wide spread of false information, such as fake news, which are harmful for society and people.

Fake news are low quality information with purposefully false data, propagated by individuals or bots that deliberately manipulate message for tattle or political plans. Some authors claim that the term "fake news" originated in previous centuries together with the mass media itself [5]. Nevertheless, this term attracted increased attention after the US presidential elections of 2016, when propagation of fake news on social media pulled attention of a larger number of online users than traditional news readers. In last five months before the elections, approximately 7.5 million tweets contained link to exceptionally one-sided or false news websites. An interesting and worrying aspect is that false and unsubstantiated news from doubtful sources attract more audiences than credible information [6]. A relevant work on this topic concluded that fake news spread quicker, penetrate further and have a deeper impact than true news [7]. There are numerous cases where people accept and spread news without checking their correctness certified by sources. By doing this, they become part of a group that deliberately or unintentionally propagates fake news. The intention behind proliferation of fake news may be manipulation of public views for financial or political benefit, or simply fun. The negative consequences of this phenomenon are therefore undeniable, ranging from wrong decision-making to episodes of bullying and violence. Figures 1a and 1b show two common examples of fake news over social networks.

False information categories are fake news, satire, misinformation, rumour, hoax, disinformation, propaganda, and opinion spam [8]. These categories are not mutually exclusive, but many researchers used them with different story lines. Although, there exist a few websites to check the authenticity of the news like PolitiFact [9], The Washington Post Fact Checker [10], FactCheck [11], Snopes [12], TruthOrFiction [13], FullFact [14], HoaxSlayer [15], Vishvas News [16], Factly Media \& Research [17] yet, these websites are unable to spontaneously react to any fake news event [18].

As online social networks are major sources of information that can mislead individuals or communities [19], there is a serious need for solutions to verify the authenticity of content. Many researchers consistently try to develop machine learning (ML) models with different sets of features targeted towards automating the fake news detection process [20][21] using visual [22] or text-based linguistic approaches. However, the following four questions remain unanswered:

- Which linguistic features are most significant in classifying 


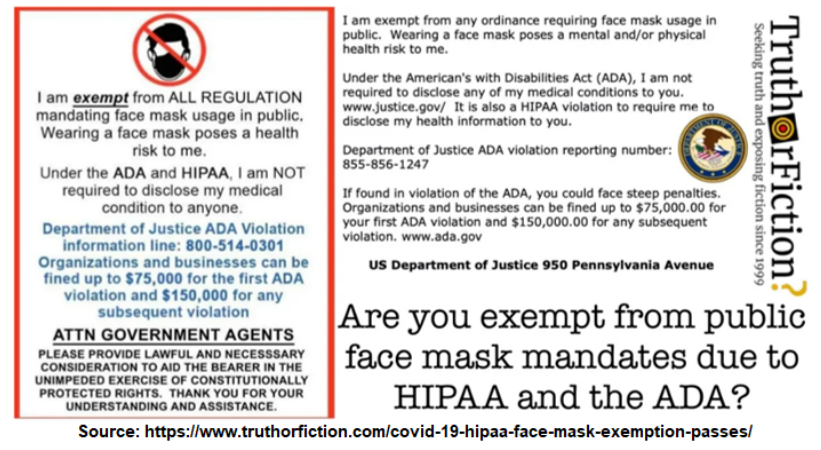

(a) Decontextualized news.

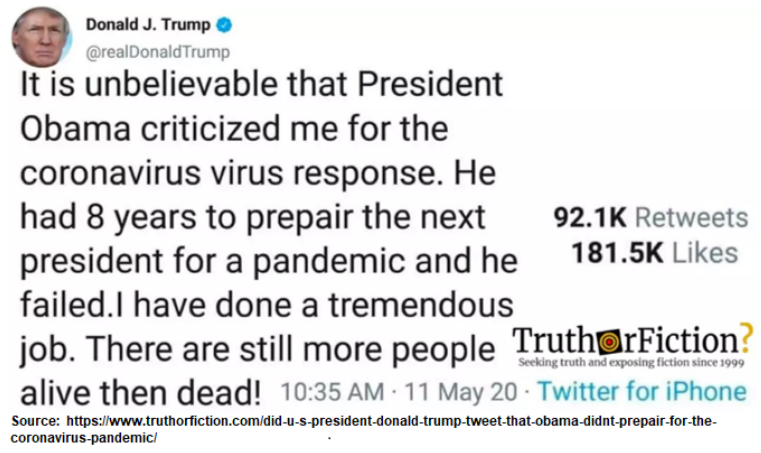

(b) False news.

Fig. 1: Fake news examples.

the news data into real and fake?

- Which WE technique with linguistic features predicts fake news better than other ML methods like convolutional neural networks (CNN) [21] or bidirectional encoder representations from transformers (BERT) [23]?

- Which classification method is the most appropriate for fake news detection on available datasets?

- Does ensemble voting classifier improve the fake news detection results?

To answer these questions, we propose a new method called WELFake exclusively focused on text data in three stages:

1) Fake news prediction using linguistic feature sets (LFS);

2) WE over LFS for improved fake news detection over a WELFake dataset.

3) Comparative analysis of the linguistic features based results with state-of-the-art CNN and BERT methods.

The WELFake model does not require additional metadata information related to the user or media [24] for the classification of real and fake news. Instead, it aims for a reformation of the state-of-art techniques in the detection of fake news over social media websites by using a combined LFS and WE technique. We highlight three contributions of our WELFake model.

a) WELFake dataset [25]: We designed a larger WELFake dataset to prevent over-fitting of classifiers and enable better ML training. For this purpose, we merged four popular news datasets (i.e. Kaggle, McIntire, Reuters, BuzzFeed Political) and prepared a more generic dataset of 72,134 news articles with 35,028 real and 37,106 fake news.

b) WELFake fake news detection model: We proposed a novel WELFake model for fake news detection in two steps: 1) collection of various linguistic features from the state-of-the-art methods and identification of a subset that performs well on the larger WELFake dataset, and 2) ensemble learning on WE features using various ML methods.

c) WELFake model generalization and validation: We applied an adversarial approach to evaluate the model generalization and effectiveness by training and testing on separate datasets.

Experimental results on the WELFake dataset revealed that our model achieved a fake news classification accuracy of up to $96.73 \%$, which improves the state-of-the-art by $4.25 \%$ over CNN and by $1.31 \%$ over BERT methods.
The paper has eight sections. Section II gives a background overview on text classification methods. Section III highlights the related work. Section IV describes the proposed methodology and the WELFake model, followed by the resulting algorithm in Section V. Section VI describes the implementation of CNN and BERT state-of-the-art methods for fake news classification used for direct comparison. Section VII provides evaluation results and further related work comparison. Section VIII concludes the paper and highlights the future work.

\section{TEXT CLASSIFICATION BACKGROUND}

This section discusses several ML [26] methods, including CNN and BERT for text classification.

\section{A. ML classification methods}

We review in this section a few ML methods [26] used for fake news classification in the WELFake model.

a) Naive Bayes $(N B)$ : is a supervised learning algorithm based on Bayes' theorem that gives fast predictions with better accuracy in the domain of sentiment analysis, spam filtration and text classification.

b) Support vector machine (SVM): is a supervised learning algorithm that works for both classification and regression problems. The algorithm finds the best line for set separation and predicts the correct set for new data values.

c) Decision tree $(D T)$ : is a supervised learning algorithm that classifies the data for both categorical and continuous dependent variables. This classifier uses tree structures to solve a problem by distributing complete datasets into homogeneous ones. Internal nodes, branches and leaf nodes in this tree structure represent the dataset, the decision rules and the outcome. There are two attribute selection measures for the best attribute node: information gain and Gini index.

d) Random forest $(R F)$ : is a supervised learning algorithm based on ensemble learning that ensembles several DTs into a RF and calculates the average results. The large number of trees in the random forest may increase the model accuracy.

e) K-nearest neighbor $(K N N)$ : is majorly useful for classification problems based on feature similarity. The algorithm can use any integer value for $K$ based on the problem statement and statistics, and employs the Euclidean, Manhattan or Hamming metric for calculating the distance between data. 
f) Boosting: connects all base learners sequentially. Initially, it passes a few records to the first base learner $\left(B L_{1}\right)$ (of any model) for training, evaluates all the records on $B L_{1}$, and passes the incorrectly classified ones to the second learner $\left(B L_{2}\right)$ for training. $B L_{2}$ tests all the records and passes the incorrectly classified ones to the next learner $B L_{3}$. This process continues until a specified number of base learners.

g) Bagging: also known as bootstrap aggregation, is an ensemble technique that uses multiple base learners and provides different subsets of the original dataset to each model for training (bootstrapping). The testing process decides the output based on the majority votes from the different models (aggregation). Apart from different sample sets, one can train the models with different subsets to reduce over-fitting.

\section{B. Text classification methods}

This section reviews the state-of-the-art CNN and BERT classification methods used in our experimental evaluation.

a) CNN [27]: splits each sentence into words (called tokens) and converts them into vectors using context-based WE methods like GloVe, Word2vec and FastText. These vectors join together to form a $m \times n$ matrix for a given sentence, where $m$ is the embedding dimension and $n$ is the number of tokens present in the vocabulary. Next, it applies the filters on the one dimensional convolution (Conv1D) layer. This convolution kernel works from top to bottom (single directional) because of the same width of filters. The Conv1D later combines its outcomes and applies pooling (maximum, average, global) that passes the data to the final fully connected layer. This takes care of the output generated by the pool layer and produces the classification decision depending on the loads appointed to each other inside the text.

b) BERT [28]: is a popular pre-trained model for text processing developed by Google, which gives better sense of the language context compared to uni-directional models in two phases: 1) bi-directional training of an input and output transformer representation model, comprising: a) an encoder reading the text as input, and b) a decoder generating the output based on the task; and 2) popular attention mechanism with a NN implementing important features only [29]. BERT takes input text and pre-processes it using tokenization, lemmatization, stopword removal and text lowering operations. Secondly, it passes the pre-processed text to the encoding phase where additional token, segment and positional embedding processes take place. Thirdly, it converts the input into default 768 long embedding vectors and passes them through the encoder layers. Finally, the more accurate information of each token available at the last encoding layer passes to the dense layer for text categorisation.

\section{STATE-OF-THE-ART SURVEY}

This section focuses on reviewing various important fake news detection methods.

1) Clickbaits: are a special type of fake content that contain linguistic headlines to attract the readers but do not fulfill their promises. Chen et al. [30] scrutinized potential techniques for programmed discovery of clickbaits by combining syntax and semantics under textual cues, as well as images and news reader behavior under non-textual cues. They surveyed the importance of these cues for the identification of fake news, but failed to implement a prototype of their methodology. Bourgonje et al. [31] proposed a model for clickbait detection that checks the relevance of headlines for article bodies. They used the dataset released by the coordinators of the first fake news challenge on stance detection and achieved a significant accuracy of $89.59 \%$ using a logistic regression classifier. Rashkin et al. [32] compared the language of real news with satire, hoaxes and propaganda, and discovered the features of fake text and other online sources for fact checking.

2) News credibility: Alrubaian et al. [33] analyzed the credibility of 489,330 Twitter accounts using text (sentiment analysis), user (gender, number of followers) and message (URL, hashtag, number of replies) specific features. They implemented RF, NB, DT and feature-rank NB algorithms with a ten-fold cross-validation. Castillo et al. [34] detected fake news based on credibility using four feature sets: textspecific (e.g., number of characters, words, question marks, punctuation, sentiment analysis), user-specific (e.g., verified accounts, number of followers, number of tweets, account creation time), message-specific (e.g., with URL, retweeted status) and propagation (e.g., root node degree, propagation tree depth). These features applied on a Twitter dataset obtained precision and recall in the range between $70 \%$ and $80 \%$. Horne et al. [35] classified the news as real or fake based on stylistic (e.g., syntax, text style, grammar), complexity (e.g., article title) and psycholinguistic features. Experimental results on three datasets revealed better results with large amounts of data and more in-depth features. Kaliyar et al.[36] proposed a deep CNN method called FNDNet to detect fake news and achieved a $98.36 \%$ accuracy on the Kaggle dataset. However, they did not show results on generalized text [37]. Shu et al. [38] discussed the worthiness of real or fake news detected through comments on particular items, and achieved $90.4 \%$, respectively $80.80 \%$ accuracy on the PolitiFact and GossipCop datasets. Zellers et al. [39] proposed a text generation model named GROVER that uses adversaries to spread more trustworthy disinformation than humans. They investigated several propagated threats and showed that GROVER can be an effective discriminator that outperforms BERT in detecting the generated false news.

3) Linguistic features: Using linguistic features for fake news detection has been popular since mid-2000s. Burgoon et al. [40] used sixteen linguistic features categorized in four classes, which achieved an accuracy of $60.72 \%$ using a DT algorithm with a 15-fold cross-validation. Vicario et al. [41] used different features like text (e.g., number of characters, words, sentences, question marks, negations), userspecific and message-specific (e.g., number of replies, likes) to identify hoaxes and fake news on social media using linear regression, logistic regression, SVM, KNN and neural networks. The validation on an Italian Facebook dataset with new features achieved an accuracy of $91 \%$ on the linear regression classification algorithm. Pérez-Rosas et al. [42] used major linguistic features (e.g., n-grams, punctuation, psycho-linguistic, readability, syntax) and achieved an accu- 
TABLE I: State-of-the-art comparison of linguistic features.

\begin{tabular}{|c|c|c|c|c|c|c|}
\hline Linguistic feature & {$[35]$} & {$[41]$} & {$[48]$} & {$[49]$} & {$[37]$} & WELFake \\
\hline Readability index & $\checkmark$ & $\boldsymbol{X}$ & $\boldsymbol{X}$ & $\boldsymbol{X}$ & $\checkmark$ & $\checkmark$ \\
\hline Psycho-linguistic & $\checkmark$ & $\boldsymbol{X}$ & $\boldsymbol{X}$ & $\checkmark$ & $\checkmark$ & $\checkmark$ \\
\hline Stylistic & $\checkmark$ & $\checkmark$ & $\boldsymbol{X}$ & $\boldsymbol{X}$ & $\checkmark$ & $\checkmark$ \\
\hline User credibility & $\boldsymbol{X}$ & $\checkmark$ & $\boldsymbol{X}$ & $\checkmark$ & $\boldsymbol{X}$ & $\boldsymbol{X}$ \\
\hline Quantity & $\boldsymbol{X}$ & $\boldsymbol{X}$ & $\boldsymbol{X}$ & $\boldsymbol{X}$ & $\checkmark$ & $\checkmark$ \\
\hline
\end{tabular}

TABLE II: Fake news detection datasets.

\begin{tabular}{|c|c|c|}
\hline Dataset & Articles & Categories \\
\hline Benjamin Political News [35] & 225 stories & Real, fake, satire \\
\hline Burfoot Satire News [50] & 4,233 news & Real, satire \\
\hline BuzzFeed News [35] & 101 news & Real, fake \\
\hline CREDBANK [44] & 60 million tweets & Real, fake \\
\hline Fake News Challenge [51] & 50,000 articles & $\begin{array}{c}\text { Agree, disagree, } \\
\text { discuss, unrelated }\end{array}$ \\
\hline FakeNewsNet [49] & 422 news & Real, fake \\
\hline LIAR [52] & 12,800 news & $\begin{array}{c}\text { True (mostly, half, } \\
\text { barely), false, pants-fire }\end{array}$ \\
\hline Reuters [48] & 44,898 articles & Real, fake \\
\hline McIntire [53] & 6,335 news & Real, fake \\
\hline Kaggle [54] & 38,729 news & Real, fake \\
\hline
\end{tabular}

racy of $76 \%$ on two novel datasets covering seven domains. Buntain et al. [43] attempted to classify real or fake Twitter threads in four categories using 45 features: structural (e.g., length, number of tweets), content (e.g., polarity, subjectivity), temporal and user (e.g., age, followers, authenticity). They used the CREDBANK [44] and PHEME [45] datasets for training and the BuzzFeed dataset for testing with an accuracy of $65.29 \%$. Newman et al. [46] applied logistic regression to evaluate 29 features and achieved an accuracy between $52 \%$ and $67 \%$. Similarly, Zhou et al. [47] used 20 features of nine categories for fake news detection. Ahmed et al. [48] used an unclear private version of the continuously updated Kaggle dataset for fake news detection and achieved an accuracy of 92\%. Shu et al. [49] used Politifact and BuzzFeed datasets in their analysis, which are hard to generalise due to their very small size. Gravanis et al. [37] compared their model with [48], [49] by combining some articles from four datasets (Kaggle-EXT, McIntire, BuzzFeed, and Politifact) in a new UNBiased dataset with 3,004 articles and achieved a highest accuracy of $95 \%$ using SVM. Table I shows a comparative study of five linguistic feature categories.

a) Readability index: quantifies the text's complexity (i.e., read difficulty) based on word length, number of syllable and sentence length.

b) Psycho-linguistic: features describe emotions, behaviours, persona, and mindset.

c) Stylistic: features explain the style of a sentence.

d) User credibility: features describe user information.

e) Quantity: features explain sentence information such as number of words and number of sentences.

4) Open datasets: From the large number of datasets available for the study of fake news [55], [56], [57], [58], [59], [60], [61], we highlight some popular datasets in Table II.

a) Benjamin Political News: is a very small dataset with 75 stories each for real, fake and satire categories.

b) Burfoot Satire News: is an unbalanced dataset with real and satire categories. c) BuzzFeed News: is a very small dataset of 101 news.

d) CREDBANK: consists of incomplete news articles in real and fake events categories.

e) Fake News Challenge: focuses on individual claims among three categories: disagree, discuss and unrelated.

f) FakeNewsNet: consists of only 422 news articles with incomplete classification in real and fake categories.

g) LIAR: contains various hard to classify social media posts and speeches due to the lack of verification sources or knowledge bases [62].

h) Reuters: consists of real and fake news articles from a single source that increases the chances of biased data.

i) McIntire: consists of real and fake news categories without authentic confirmation from any individual.

j) Kaggle: consists of real and fake news data without source information.

5) Summary: No single method guarantees the best solution for all datasets. The state-of-the-art approach of Gravanis et al. [37] used 57 linguistic features and embed them with word-to-vector embedding method on UNBiased dataset of less than 4000 articles only. They used all features in single LFS and achieves up to $95 \%$ accuracy. WELFake improves on this method using a novel method based on four stages that: 1) creates a larger dataset with improved generalization; 2) identifies the most significant twenty linguistic features and creates three unique LFS based on categories; 3) applies two WE methods to train various ML models; and 4) generates the final prediction using a two-stage voting classification.

\section{WELFAKE MODEL}

This section outlines the WELFake model for fake news detection divided in four phases, shown in Figure 2.

1) Dataset preparation involves collection and preprocessing of the data in a proper format, as a fundamental task of any ML model;

2) Feature engineering involves linguistic feature extraction and selection;

3) WE identifies the most appropriate technique connected with the LFS;

4) Fake news detection tunes the model parameters and applies hard voting classifier for better accuracy.

\section{A. Dataset preparation}

1) News data collection: is essential for a balanced and unbiased dataset and the key for providing high quality training data and delivering good results. Although there exist an important number of open datasets for the study of fake news (see Section III and Table II), the literature showed their serious limitations in terms of size, category or bias. After a careful study, we prepared a more comprehensive WELFake dataset that combines four datasets, Kaggle, McIntire, Reuters and BuzzFeed, for two reasons. Firstly, they have a similar structure with two categories (i.e., real and fake news). Secondly, combining the datasets reduces the limitations and the bias of each individual dataset. Table III shows the WELFake open dataset with 72,134 news articles classified as 35,028 real and 37,106 fake news [25]. The dataset contains three 


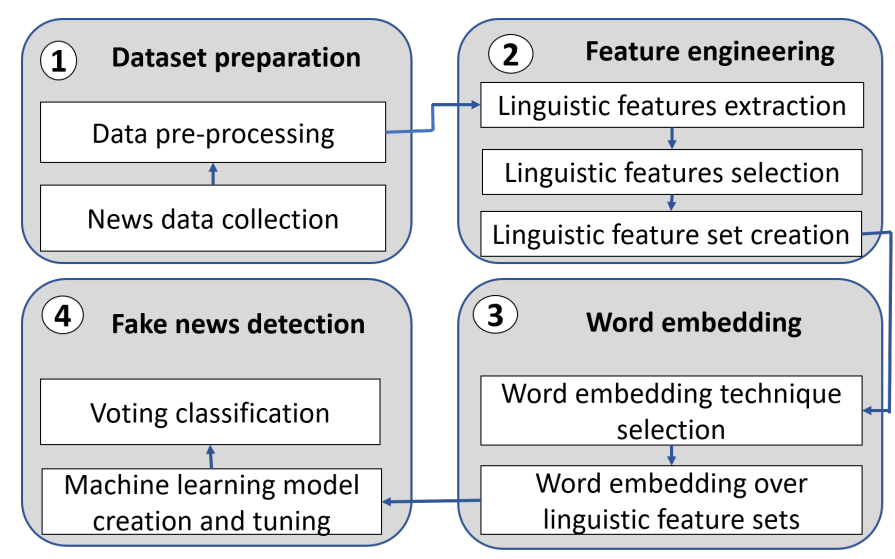

Fig. 2: WELFake model overview.

TABLE III: WELFake dataset.

\begin{tabular}{|c|c|c|}
\hline Dataset & Real news & Fake news \\
\hline Kaggle & 10387 & 10413 \\
\hline McIntire & 3171 & 3164 \\
\hline Reuters & 21417 & 23481 \\
\hline BuzzFeed Political & 53 & 48 \\
\hline WELFake dataset & $\mathbf{3 5 , 0 2 8}$ & $\mathbf{3 7 , 1 0 6}$ \\
\hline
\end{tabular}

columns (i.e., title, text, label) with a binary label for fake and real news. Table IV summarises the balanced fake and real news distribution in the WELFake dataset across all four feature categories.

a) Number of short sentences: (below ten words) representing real news is greater than those representing fake news;

b) Text readability: of fake news is poorer than the readability of real news;

c) Subjectivity: of fake news articles is larger than for real news articles;

d) Number of articles: representing real news is larger than those representing fake news.

2) Data pre-processing: solves different problems in the collected data, like typographic errors, unstructured data format and other limitations, using several methods, depending on the dataset and objectives.

a) Missing data: handles undefined $(\mathrm{NaN})$ and blank values $(N U L L)$ present in the dataset, which hinder the feature engineering process. Since deleting the data entries containing missing values may cause the loss of important information [63], we performed a missing value imputation process that estimates missing values and then analyzes the complete data set as if these values were the actual observed ones.

b) Inconsistent data: deviates from other data points because of mistakes during data collection. We employed several visualization techniques and mathematical functions for outlier identification and correction, like box plot, scatter plot, Z-score, IQR score.

c) Duplicate data: or de-duplication removes redundancy that can lead to biased results, which may occurs when same person collected the data.

d) Irrelevant data: removes stop words (and other noise) that make the sentence grammatically complete, but do not have semantic significance in news classification operations.
TABLE IV: Fake versus real news distribution in WELFake.

\begin{tabular}{|c|c|c|}
\hline Category & Real news [\%] & Fake news [\%] \\
\hline Short sentences & 60.9 & 39.1 \\
\hline Readability index & 51.7 & 48.3 \\
\hline Subjectivity & 45.4 & 54.6 \\
\hline Number of articles & 53.9 & 46.1 \\
\hline WELFake dataset & $\mathbf{4 8 . 5 5 \%}$ & $\mathbf{5 1 . 4 5 \%}$ \\
\hline
\end{tabular}

Removing stop words and keeping relevant tokens only significantly increases the model performance.

e) Stemming: converts the text into its root word by applying the Porter-Stemmer algorithm on text features for accuracy improvement. In case it cannot recognize the root word, it generates the canonical form of a corresponding word.

\section{B. Feature engineering}

1) Linguistic features extraction: is the process of conversion from raw text to the data provided to the ML algorithm. Feature extraction aims to create a feature set that summarizes the information of the original dataset, which speeds up the model training and improves the data visualization and learning accuracy. We extracted in WELFake 87 text-based linguistic features from state-of-the-art works, falling in two syntactic (i.e., writing pattern, quantity) and semantic (i.e., grammar, psycho-linguistics) categories.

a) Writing pattern: emphasises the writing style of the text by accessing the sentence types, use of determinants, special characters, and modifiers.

b) Grammar: focuses on the text readability index and emphasises the word structure, average syllables per word, easy word use ratio in a word list, and sentence complexity.

c) Psycho-linguistic: estimates the text sentiment and information opinion based on semantic and subjectivity.

d) Quantity: identifies speech information parts by counting the verbs, adjectives, adverbs, syllables and words, as well as the rate of adjectives, adverbs and words per sentence.

2) Linguistic features selection: chooses important features for data classification, which diminishes the quantity of input features, decreases the computational expense and improves the accuracy of the ML model. For this purpose, we calculated the Pearson's correlation coefficient of each feature with the other features within the same category and discarded those with a correlation coefficient higher than 0.7 , which indicates a strong positive linear relationship [23] between the two features. A learning model with fewer features is simpler and more precise according to Occam's razor principle and the minimum description length concept [62]. We executed this recurrent process until removing all least significant features. Table $\mathrm{V}$ shows the remaining twenty most significant features of WELFake grouped based on the four categories.

3) LFS creation: groups the selected twenty linguistic features in different sets to enable multiple WE methods for an unbiased model training. We need an odd number of input sets to obtain a clear result upon subsequent voting classification. We consequently created a minimum of three distinct LFS based on four categories, as shown in Table V. 
a) Readability index: defines the sentence structure complexity of any text. We identify the level or grade of the text writer based on the readability index, which helps in classifying the news as real or fake. For this reason, we uniformly distributed all three readability index features among the three LFS and assigned one feature to each set.

b) Psycho-linguistic: features play important role in detection of fake news, as explained in Section III. We therefore used all three of them in all the three LFS.

c) Quantity: features also participate in news classification, so we evenly distributed them across the three LFS.

d) Writing pattern: contains five features and therefore, we evenly distributed three features to each LFS.

\section{WE}

1) WE selection: identifies the most appropriate technique for converting plain text into a numeric value, as ML methods cannot directly process plain text. We observed two popular WE categories in the literature: 1) content-based, such as TF-IDF and CV, that focus on previous knowledge, and 2) context-based, such as Word2Vec, GloVe and FastText, that focus on writing text patterns. As fake news writers (fakesters) tend to repeat similar words, we selected the content-based WE that focuses on writing patterns rather than context [64].

a) Count vectorizer $(\mathrm{CV})$ : also called one-hot encoding, converts text document in a histogram vector, where each element represents the number of appearances of the word in the document. The vector length depends on the number of unique words in the corpus.

b) Term frequency-inverse document frequency $(T F-I D F):$ is the advanced version of $\mathrm{CV}$, which shows the importance of a term (representing a word) in a corpus alongside its occurrence in the document. $T F-I D F$ is the multiplication of the term frequency $t f(t, d)$ that computes the occurrence of a term $t$ in a document $d$ and the inverse document frequency $i d f(t, D)$ that computes the importance of that term $t$ in a corpus of documents $D$ :

$$
\begin{aligned}
T F-I D F(t, d, D) & =t f(t, d) \cdot i d f(t, D) ; \\
t f(t, d) & =\frac{f_{d}(t)}{\max _{w \in d} f_{d}(w)} ; \\
i d f(t, D) & =\ln \left(\frac{|D|}{|\{d \in D: t \in d\}|}\right),
\end{aligned}
$$

where $f_{d}(t)$ represents the number of occurrences of the term $t$ in the document $d,|D|$ is the number of documents in the corpus $D$, and $|\{d \in D: t \in d\}|$ is the number of documents containing the term $t$.

2) WE over LFS: improves the output prediction, as predefined features do not always accurately predict and need additional training methods. For this purpose, we combined the WE with LFS. We applied the TF-IDF and CV WE techniques on the three LFS and found that CV gives better results. We achieved maximum $95.61 \%$ accuracy using SVM on CV, while TF-IDF gave maximum accuracy of $95.12 \%$ using bagging. Thus, we selected CV and combined it with LFSs for further

\begin{tabular}{|c|c|c|c|c|}
\hline Category & Feature & LFS1 & LFS2 & LFS3 \\
\hline \multirow{5}{*}{$\begin{array}{l}\text { Writing } \\
\text { pattern }\end{array}$} & Number of special characters & $\checkmark$ & $x$ & $\checkmark$ \\
\hline & Number of determinants & $x$ & $\checkmark$ & $\checkmark$ \\
\hline & Number of capital letters & $x$ & $x$ & $\checkmark$ \\
\hline & Number of short sentences & $\checkmark$ & $\checkmark$ & $x$ \\
\hline & Number of long sentences & $\checkmark$ & $\checkmark$ & $x$ \\
\hline \multirow{3}{*}{$\begin{array}{c}\text { Grammar } \\
\text { (readability } \\
\text { index) }\end{array}$} & Gunning fog grade readability index & $\checkmark$ & $x$ & $x$ \\
\hline & SMOG readability index & $x$ & $\checkmark$ & $x$ \\
\hline & Automatic readability index & $x$ & $x$ & $\checkmark$ \\
\hline \multirow{3}{*}{$\begin{array}{c}\text { Psycho- } \\
\text { linguistics }\end{array}$} & Text polarity & $\checkmark$ & $\checkmark$ & $\checkmark$ \\
\hline & Title similarity & $\checkmark$ & $\checkmark$ & $\checkmark$ \\
\hline & Subjectivity & $\checkmark$ & $\checkmark$ & $\checkmark$ \\
\hline \multirow{9}{*}{ Quantity } & Number of syllables & $x$ & $x$ & $\checkmark$ \\
\hline & Number of words & $x$ & $\checkmark$ & $x$ \\
\hline & Rate of adjectives and adverbs & $\checkmark$ & $x$ & $x$ \\
\hline & Words per sentence & $x$ & $x$ & $\checkmark$ \\
\hline & Number of articles & $\checkmark$ & $x$ & $x$ \\
\hline & Number of verbs & $x$ & $\checkmark$ & $x$ \\
\hline & Number of sentences & $x$ & $\checkmark$ & $x$ \\
\hline & Number of adjectives & $\checkmark$ & $x$ & $x$ \\
\hline & Number of adverbs & $x$ & $x$ & $\sqrt{ }$ \\
\hline
\end{tabular}
accuracy analysis of various models in Section VII-B.
TABLE V: WELFake LFS.

\begin{tabular}{|c|c|c|c|c|}
\hline Model & Iterations & Hyper-parameter & Value range & Value \\
\hline \multirow{2}{*}{ NB } & \multirow{2}{*}{4} & Smoothing & {$[0,1]$} & 1 \\
\hline & & Fit prior & $\{$ true, false $\}$ & true \\
\hline \multirow{4}{*}{ SVM } & \multirow{4}{*}{9} & Kernel & $\begin{array}{c}\{\text { linear, poly, rbf, } \\
\text { sigmoid, precomputed }\}\end{array}$ & linear \\
\hline & & Regularization & $\mathbb{R}$ & 100 \\
\hline & & Kernel coefficient & $\begin{array}{c}\{\text { float }, \\
x \mid x \in\{\text { scale, auto }\}\}\end{array}$ & 0.0001 \\
\hline & & Degree & $\mathbb{Z}$ & 3 \\
\hline \multirow{2}{*}{ KNN } & \multirow{2}{*}{5} & Number of neighbors & $\mathbb{Z}$ & 2 \\
\hline & & Weights & uniform, distance, callable & uniform \\
\hline \multirow{3}{*}{ DT } & \multirow{3}{*}{8} & $\begin{array}{c}\text { Maximum number } \\
\text { of features }\end{array}$ & $\begin{array}{c}\{\text { integer, float, none, } \\
x \mid x \in\{\text { auto, sqrt, } \log 2\}\}\end{array}$ & none \\
\hline & & \begin{tabular}{|c|} 
Criterion \\
\end{tabular} & $\{$ gini, entropy $\}$ & gini \\
\hline & & Cost complexity pruning & $\mathbb{R}_{+}$ & 0.02 \\
\hline \multirow{3}{*}{ Bagging } & \multirow{3}{*}{6} & Base estimator & $\{$ object, none $\}$ & none \\
\hline & & Number of estimators & $\mathbb{Z}$ & 100 \\
\hline & & Bootstrap & true, false $\}$ & true \\
\hline \multirow{2}{*}{ AdaBoost } & \multirow{2}{*}{5} & Number of estimators & $\mathbb{Z}$ & 50 \\
\hline & & Learning rate & {$[0,1]$} & 1 \\
\hline
\end{tabular}

TABLE VI: Hyper-parameters for ML models tuning.

\section{Fake news detection}

1) ML model creation and tuning: passes the LFS with WE through six ML methods: SVM, NB, KNN, DT, Bagging and AdaBoost. For this purpose, we experimented each ML model on random samples of the WELFake dataset with four trainingtesting data combinations: $60 \%-40 \%, 70 \%-30 \%, 80 \%-$ $20 \%$ and $90 \%-10 \%$. To improve the accuracy, we performed a manual tuning of the six different models using the hyperparameters displayed in Table VI. We sequentially explored different hyper-parameter value combinations from the given possible value ranges and tuned them until we obtained a state-of-the-art accuracy of at least $96 \%$. We evaluated the performance of each ML model on different training and testing data distributions as shown in Table $\mathrm{X}$ in Section VII-B and found out that a $70 \%-30 \%$ data distribution gives better accuracy for all six ML methods.

2) Voting classification: uses ensemble learning to collect predictive outputs from various models and generates an output that minimises the error and the over-fitting. There are in general two voting classifier approaches: soft voting based on probability and hard voting based on maximum votes. Since fake news detection is a binary classification problem, we use 


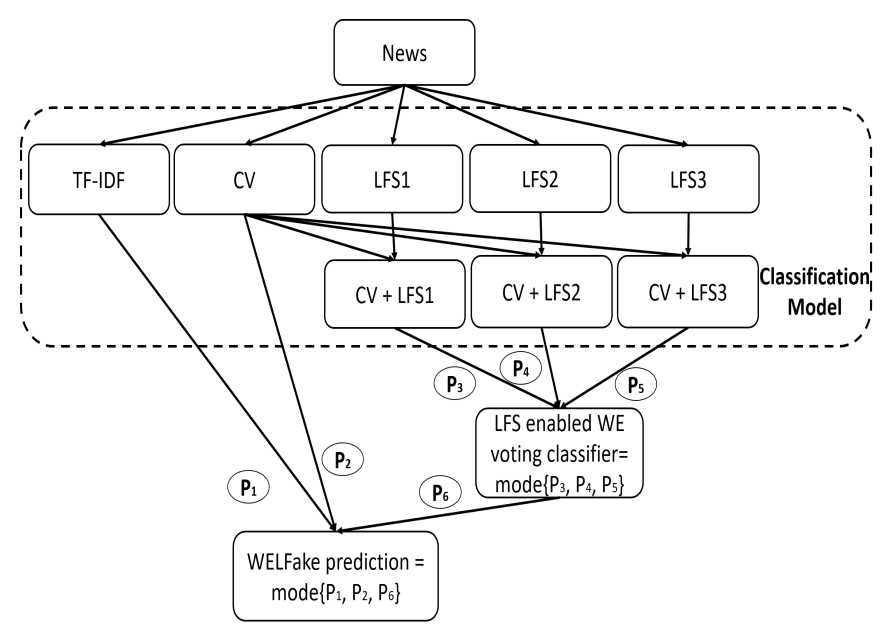

Fig. 3: Sequential workflow of WELFake model.

hard voting that predicts a target variable $Y$ based on the maximum votes mode given by different models $M_{i}$ to a class:

$$
Y=\operatorname{mode}\left\{M_{1}(X), M_{2}(X), \ldots, M_{n}(X)\right\},
$$

where $X$ is predictor or input variable.

\section{Welfake Fake News Detection Algorithm}

Figure 3 shows the WELFake binary news classification method (as real or fake) using TF-IDF and CV, three linguistic feature sets (LFS1, LFS2 and LFS3) and the hard voting classifier. The WELFake workflow consists of four steps:

1) Apply TF-IDF and CV on entire dataset, store the results in $P_{1}$ and $P_{2}$ and decide the better WE based on accuracy.

2) Apply CV (better performing method) on three welldefined LFS and store the results in $P_{3}, P_{4}$, and $P_{5}$.

3) Apply WE hard voting classifier on $P_{3}, P_{4}$ and $P_{5}$ and generate the prediction $P_{6}$ as output.

4) Combine $P_{1}, P_{2}$ and $P_{6}$ using the hard voting classifier and generate the final prediction output.

Algorithm 1 explains the steps of proposed WELFake model organized in four phases, as explained in Figure 2.

a) Dataset preparation: covers the WELFake data collection in line 1 and dataset pre-processing in line 2.

b) Feature engineering: extracts linguistic features of the dataset in line 3 and applies Pearson's coefficient to select the significant linguistic features in line 4 . Line 5 creates an odd number of LFS to perform voting.

c) WE: (i.e., $\mathrm{CV}$ and TF-IDF) applies on the entire dataset in lines 6 and 7. We select in line 8 the best method to combine with the various LFS created in line 5. Lines 9 and 10 combine the LFS with best WE method.

d) ML model tuning and voting classifier: We train in line 11 the datasets from line 5 on various ML classification models and select the best results from each set. Line 12 applies the voting classifier on the results achieved on the different LFS using the best ML classification model and generates the hard voting output. Line 13 applies again the hard voting classifier (line 12), CV (line 6) and TF-IDF (line 7 ) and returns the final news classification prediction.

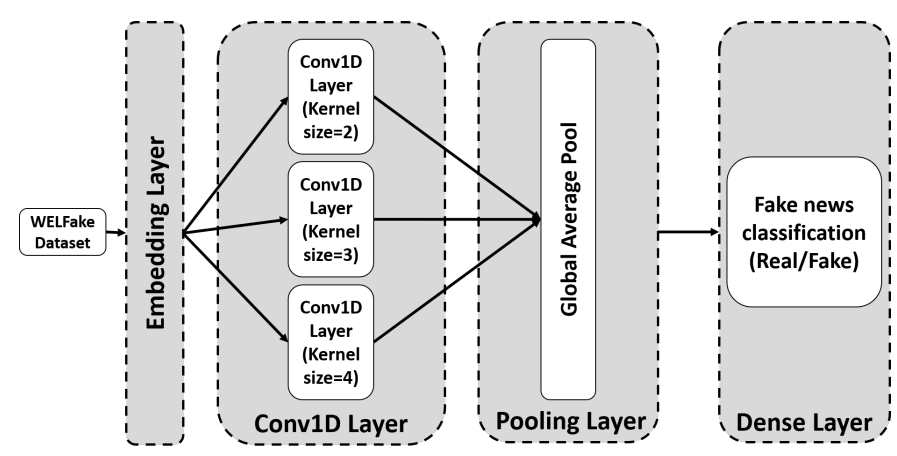

Fig. 4: CNN text processing on WELFake dataset.

\section{CNN AND BERT IMPLEMENTATIONS ON WELFAKE DATASET}

This section describes the CNN and BERT implementation on the WELFake dataset. We performed a hyper-parameter tuning to improve their performance, summarized in Table VII.

\section{A. CNN-based fake news detection}

After preprocessing the text as presented in Section IV-A2, we pass it to our CNN implementation consisting of several layer shown in Figure 4.

a) Embedding layer: converts the input text into WE, where every word in the vocabulary transforms into a highdimensional space vector for next layer execution. We used the GloVe technique for conversion of text into vector.

b) Convolutional layer: takes the WE from the previous layer as input and specifies the number of Conv1D layers and the kernel size $k$, representing the number of words in a sentence during one filter slide. We used three Conv1D layers having kernels of size two, three and four with 32 filters each (totalling 96 filters in the Conv1D layers). We obtained 32 feature maps from each kernel for further execution and calculated the filter height as $m-k+1$, where $m$ is maximum sequence length in the dataset.

c) Pooling layer: reduces the number of outputs generated at the previous layer. After applying a global average pooling, the size of the vector is same as the number of filters. This layer also merges the converted vectors of size $f \times x$, where $f$ is number of filters and $x$ is number of kernels.

d) Dense layer: takes the output from pooling layer and passes it to two consecutive dense layers for final prediction using a Sigmoid activation function with values in the $[0,1]$ interval, which helps assessing the $\mathrm{CNN}$ prediction confidence.

\section{B. BERT-based fake news detection}

We performed fine tuning of the pre-trained BERT model for fake news classification in several steps, shown in Figure 5.

a) Data pre-processing: performs the operations described in Section IV-A2 on the raw text and adds a classification [CLS] token at the start of the first sentence. 


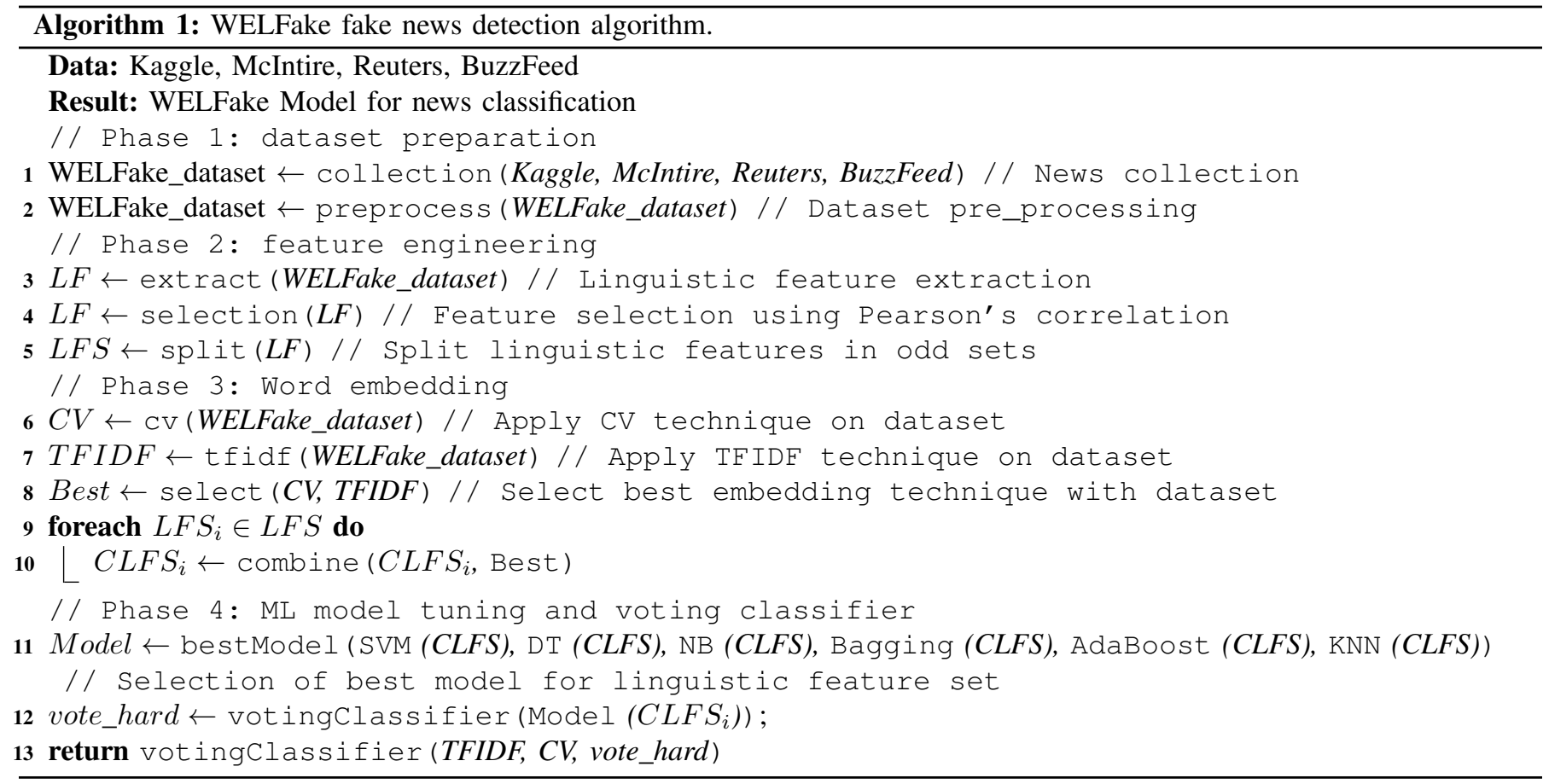

TABLE VII: CNN and BERT model parameters.

\begin{tabular}{|c|c|c|}
\hline Model & Parameter & Value \\
\hline \multirow{4}{*}{ CNN } & Number of Conv1D layers & 3 \\
& Number of filters & 32 \\
& Kernel Size & $2,3,4$ \\
& Aumber of global avgpool layer & 3 \\
& Optivation function & Sigmoid function \\
& Loss function & Adam \\
BERT & Number of dense layers & Binary-crossentropy \\
& Number of droupout layer & 3 \\
& Activation function & Sigmoid function \\
\hline
\end{tabular}

b) BERT model architecture: uses the BERT $B A S E$ model with 12 encoder layers. Each encoder layer performs a self-attention mechanism and passes the output to a feedforward network. The output of each layer is a vector of default size 768 . We applied at the $12^{\text {th }}$ layer the [CLS] token to store the useful information for classification.

c) Output layer: reads the vector stored in the [CLS] token. We used a Sigmoid activation function and implemented a NN with five dense layers and three dropout layers to classify the text news. The Sigmoid function produces the results in the range of $[0,1]$, which identifies confidence classes such that the class with a larger value wins the prediction.

\section{EXPERIMENTAL EVALUATION}

We implemented WELFake in Python 3.6 on a Jupyter notebook. We run the experiments on a computer equipped with a ninth generation i7 processor and $16 \mathrm{~GB}$ of memory.

\section{A. Evaluation metrics}

We define four evaluation parameters, true positive (TP), true negative (TN), false positive (FP) and false negative $(\mathrm{FN})$, based on the relation between the predictive news

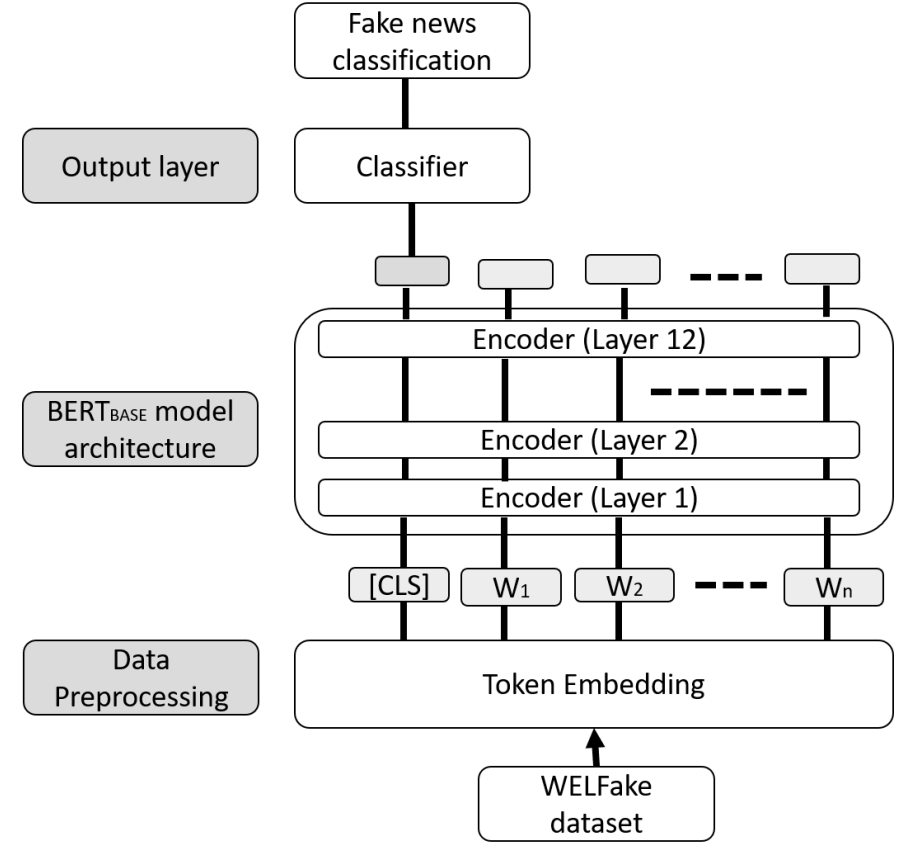

Fig. 5: BERT text processing on WELFake dataset.

TABLE VIII: Fake news prediction parameters.

\begin{tabular}{|c|c|c|}
\hline Evaluation parameter & Predictive value & Actual value \\
\hline True positive (TP) & Yes & Yes \\
True negative (TN) & No & No \\
False positive (FP) & Yes & No \\
False negative (FN) & No & Yes \\
\hline
\end{tabular}

classification and the actual one, displayed in Table VIII. Based on these parameters, we evaluated WELFake model on four performance metrics. 
a) Accuracy: is the ratio between the number of correct predictions and the total number of predictions:

$$
\text { Accuracy }=\frac{T P+T N}{T P+T N+F P+F N} .
$$

b) Precision: measures the positive predicted value, as the ratio between the number of correct positive predictions to the total number of positive predictions:

$$
\text { Precision }=\frac{T P}{T P+F P} .
$$

c) Recall: $R$ measures the sensitivity of the model as the ratio between the number of correct positive predictions to the total number of correctly predicted results:

$$
\text { Recall }=\frac{T P}{T P+F N} .
$$

d) F1-score: F1 measures the testing accuracy of the model as the harmonic mean of the precision and the recall:

$$
F 1-\text { score }=\frac{2}{\text { Recall }^{-1}+\text { Precision }^{-1}} .
$$

\section{B. Comparative ML classification}

We analyse the accuracy of the WELFake model on the six ML classifiers in Table IX using four scenarios.

a) LFS: classifies the news on the three LFS individually using the six ML classifiers. The accuracy of all three sets ranges between $77.3 \%$ and $85.6 \%$. LFS1 gives the best accuracy of $83.4 \%$ using Bagging and the worst accuracy of $79.2 \%$ using NB. LFS2 produces the highest accuracy of $84.2 \%$ using Bagging and the lowest accuracy of $77.3 \%$ using NB. Similarly, LFS3 achieves the highest $85.6 \%$ accuracy using SVM and the lowest accuracy of $79.8 \%$ using NB. Bagging and SVM performed the best among all six ML classifiers followed by AdaBoost, DT, KNN and NB.

b) WE: applies CV and TF-IDF on the WELFake dataset and classifies the news to predict $P_{1}$ and $P_{2}$, as illustrated in Figure 3. We observed that $\mathrm{CV}$ achieved in general a better accuracy than TF-IDF. CV performed the best on SVM with a $95.61 \%$ accuracy, followed by Bagging, AdaBoost, NB, DT and KNN. TF-IDF achieved an accuracy of $95.12 \%$ using Bagging, followed by SVM, AdaBoost, NB, KNN and DT.

c) LFS-enabled WE: combines CV with the three LFS to predict $P_{3}, P_{4}$ and $P_{5}$ and applies voting classifier to obtain $P_{6}$. We achieved a maximum accuracy of up to $96.1 \%$ using SVM and a minimum accuracy of up to $89.6 \%$ using DT. Overall, SVM performed the best followed by Bagging, AdaBoost, NB, KNN and DT.

d) WELFake prediction: predicts the final classification output by applying voting classifier across the LFS-enabled WE classification $\left(P_{6}\right)$, TF-IDF $\left(P_{1}\right)$ and $\mathrm{CV}\left(P_{2}\right)$ predictions. SVM achieved the maximum accuracy of $96.73 \%$ followed by AdaBoost, Bagging, NB, KNN and DT.

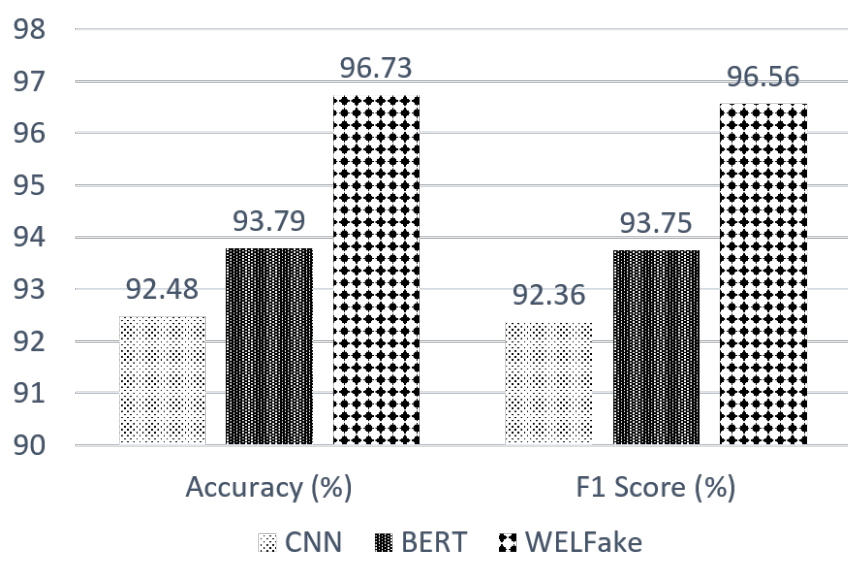

Fig. 6: CNN, BERT and WELFake performance comparison.

e) Comparison: Table XI compares the overall performance of the WELFake model in terms of accuracy, precision, recall and F1-score, introduced in Section VII-A. From all six ML models, WELFake produces the best results using SVM with a maximum accuracy of $96.73 \%$, and a minimum accuracy $89.92 \%$ using DT. Similarly, SVM achieved the highest precision $(94.6 \%)$, recall $(98.61 \%)$ and F-1 score $(96.56 \%)$, while DT scored the lowest precision $(86.1 \%)$ and F1-score (89.24\%). KNN achieved the worst recall of $90.55 \%$ only. From these results, we conclude that the proposed WELFake model achieved the best performance on the WELFake dataset (on all evaluation metrics) using SVM followed by AdaBoost, Bagging, NB, KNN and DT.

\section{Comparative text classification}

Figure 6 compares the accuracy and F1-score of the WELFake model with the CNN and BERT state-of-the-art methods. The WELFake model achieved a $96.73 \%$ accuracy, while CNN and BERT achieved a maximum accuracy only up to $92.48 \%$ and $93.79 \%$, respectively. Similarly, WELFake also shows a better F1-score compared to CNN and BERT due to its better generalization. While Kaliyar et al. [36] achieved a $98.36 \%$ accuracy using a deep neural network on single dataset, its accuracy reduced to $92.48 \%$ on the WELFake dataset. Similarly, BERT is a pre-trained model which works well with labeled data, while its performance gets compromised in a generalized dataset where testing data is independent from the training data. Finally, WELFake focuses on text writing pattern linguistic features that extract the text structure and provide the syntax, sentiment, grammatical and readability evidences specific to the text content, which explains the improved fake news detection. Overall, WELFake achieved the highest accuracy followed by BERT and CNN.

\section{WELFake generalization}

We use different training and testing datasets to analyze the generalization performance of the WELFake model. For this purpose, we followed an adversarial approach that splits the WELFake dataset into four constituent subsets (i.e. BuzzFeed, 
TABLE IX: Accuracy analysis of WELFake model.

\begin{tabular}{|c|c|c|c|c|c|c|c|c|c|}
\hline$\stackrel{\text { Scenarios }}{\longrightarrow}$ & \multicolumn{3}{|c|}{$L F S$} & \multicolumn{2}{|l|}{$W E$} & \multicolumn{3}{|c|}{ LFSWE } & Final prediction \\
\hline$\downarrow$ Model & LFS1 (\%) & LFS2 (\%) & LFS3 (\%) & $T F-I D F(\%)$ & $C V(\%)$ & $C V+L F S 1(\%)$ & $C V+L F S 2(\%)$ & $C V+L F S 3(\%)$ & Voting classifier $(\%)$ \\
\hline KNN & 79.6 & 81.8 & 80.6 & 89.6 & 88.2 & 90.3 & 90.5 & 90.1 & 90.16 \\
\hline SVM & 82.5 & 83.5 & 85.6 & 94.5 & 95.61 & 95.6 & 96.1 & 95.01 & 96.73 \\
\hline NB & 79.2 & 77.3 & 79.8 & 91.02 & 91.03 & 91.05 & 91.08 & 92.01 & 92.12 \\
\hline DT & 81.4 & 79.6 & 80.8 & 89.54 & 89.51 & 90.1 & 89.61 & 89.68 & 89.92 \\
\hline Bagging & 83.4 & 84.2 & 84.2 & 95.12 & 95.04 & 95.08 & 95.3 & 95.3 & 95.31 \\
\hline Adaboost & 81.8 & 81.9 & 80.6 & 93.78 & 94.9 & 95.18 & 95.18 & 95.2 & 95.32 \\
\hline
\end{tabular}

TABLE X: WELFake accuracy analysis on different training and testing datasets.

\begin{tabular}{|c|c|c|c|c|c|c|}
\hline \multirow{2}{*}{$\begin{array}{c}\text { Training-testing } \\
\text { data ratios }(\%)\end{array}$} & \multicolumn{7}{|c|}{ Model } \\
\cline { 2 - 7 } & $K N N$ & $S V M$ & $N B$ & $D T$ & Bagging & Adaboost \\
\hline $60-40$ & 89.64 & 95.23 & 92.01 & 88.97 & 95.30 & 95.12 \\
\hline $\mathbf{7 0}-\mathbf{3 0}$ & $\mathbf{9 0 . 1 6}$ & $\mathbf{9 6 . 7 3}$ & $\mathbf{9 2 . 1 2}$ & $\mathbf{8 9 . 9 2}$ & $\mathbf{9 5 . 3 1}$ & $\mathbf{9 5 . 3 2}$ \\
\hline $80-20$ & 90.16 & 96.71 & 92.12 & 89.91 & 95.32 & 95.30 \\
\hline $90-10$ & 90.15 & 96.73 & 92.12 & 89.92 & 95.30 & 95.30 \\
\hline
\end{tabular}

TABLE XI: Evaluation results for different ML models.

\begin{tabular}{|c|c|c|c|c|}
\hline Model & Accuracy (\%) & Precision (\%) & Recall (\%) & F1-Score (\%) \\
\hline KNN & 90.16 & 89.02 & 90.55 & 89.78 \\
\hline SVM & 96.73 & 94.60 & 98.61 & 96.56 \\
\hline NB & 92.12 & 91.45 & 92.25 & 91.85 \\
\hline DT & 89.92 & 86.10 & 92.62 & 89.24 \\
\hline Bagging & 95.31 & 91.78 & 98.46 & 95.00 \\
\hline Adaboost & 95.32 & 91.81 & 98.46 & 95.02 \\
\hline
\end{tabular}

Reuters, McIntire, Kaggle). We generated four experimental datasets that combine three of them as training data and keeps the forth for testing, as presented in Table XII.

a) Under-fitting dataset (3): has the training dataset smaller than the testing dataset, which overestimates the results in over-constrained models.

b) Over-fitting dataset (4): uses almost the entire dataset as part of training, which underestimates the results in underconstrained models.

c) Balanced dataset $(1,2)$ : splits the training and testing data in acceptable ratios.

The evaluation results reveal that the WELFake model accuracy remains high in all four scenarios, ranging between $96.03 \%$ to $96.84 \%$ with a very small standard deviation of 0.3 . It is possible because our proposed WELFake model handles well the overfitting and underfitting data due to the multilevel model training and data classification. These results therefore validate the generalization of proposed WELFake model.

\section{E. Related work comparison}

We compare our proposed WELFake model with three related models, summarised in Table XIII.

a) Ahmed et al. [48]: experimented fake news detection on the Kaggle-EXT dataset with 25,200 articles. They did not use linguistic features and applied linear SVM model on TFIDF with a highest $92 \%$ accuracy.

b) K. Shu et al. [49]: used linguistic features for fake news detection on the BuzzFeed and Politifact datasets of only 240 and 182 articles. They separately used a linguistic feature method with two features and implemented SVM on both datasets with an accuracy of $87.8 \%$ for Politifact and $86.4 \%$ for BuzzFeed.
TABLE XII: WELFake generalization accuracy.

\begin{tabular}{|c|c|c|c|c|c|}
\hline No. & \multicolumn{2}{|c|}{ Training dataset } & Testing dataset & Accuracy \\
\hline 1 & BuzzFeed, Reuters, McIntire & $71 \%$ & Kaggle & $29 \%$ & $96.70 \%$ \\
2 & BuzzFeed, Reuters, Kaggle & $91 \%$ & McIntire & $9 \%$ & $96.43 \%$ \\
3 & BuzzFeed, McIntire, Kaggle & $38 \%$ & Reuters & $62 \%$ & $96.72 \%$ \\
4 & Reuters, McIntire, Kaggle & $99 \%$ & BuzzFeed & $1 \%$ & $96.03 \%$ \\
\hline
\end{tabular}

TABLE XIII: WELFake comparison with related methods.

\begin{tabular}{|c|c|c|c|c|}
\hline Parameter & $\begin{array}{c}\text { Ahmed } \\
\text { et al. [48] }\end{array}$ & $\begin{array}{c}\text { Shu } \\
\text { et al. [49] }\end{array}$ & $\begin{array}{l}\text { Gravanis } \\
\text { et al. [37] }\end{array}$ & WELFake \\
\hline Dataset & 1. Kaggle & $\begin{array}{ll}\text { 1. } & \text { Politifact } \\
\text { 2. } & \text { BuzzFeed }\end{array}$ & $\begin{array}{lc}\text { 1. } & \text { Kaggle-EXT } \\
\text { 2. } & \text { BuzzFeed } \\
\text { 3. } & \text { Politifact } \\
\text { 4. } & \text { McIntire } \\
\text { 5. } & \text { UNBiased }\end{array}$ & $\begin{array}{lc}\text { 1. } & \text { Kaggle } \\
\text { 2. } & \text { BuzzFeed } \\
\text { 3. } & \text { Reuters } \\
4 & \text { McIntire } \\
5 & \text { WELFake }\end{array}$ \\
\hline $\begin{array}{l}\text { Number of } \\
\text { news articles }\end{array}$ & 1. 25,200 & $\begin{array}{ll}\text { 1. } & 240 \\
\text { 2. } & 182\end{array}$ & $\begin{array}{lr}\text { 1. } & 23,340 \\
2 . & 240 \\
3 . & 182 \\
4 . & 6,310 \\
5 . & 3,404\end{array}$ & $\begin{array}{lr}\text { 1. } & 20,800 \\
2 . & 101 \\
3 . & 44,898 \\
4 . & 6,335 \\
5 . & 72,134\end{array}$ \\
\hline Linguistic features & No & Yes & Yes & Yes \\
\hline$W E$ & TF-IDF & No & Word2Vec & $\mathrm{CV}$ \\
\hline Other features & No & No & NA & Voting classifier \\
\hline Number of features & 1 & 2 & 57 & 20 \\
\hline Classifier & Linear SVM & SVM & SVM & SVM \\
\hline Accuracy & $92 \%$ & $\begin{array}{l}\text { 1. } 87.8 \% \\
\text { 2. } 86.4 \%\end{array}$ & $\begin{array}{ll}\text { 1. } & 99.0 \% \\
\text { 2. } & 72.7 \% \\
\text { 3. } & 84.7 \% \\
\text { 4. } & 81.0 \% \\
\text { 5. } & 95.0 \%\end{array}$ & $\begin{array}{ll}1 . & 92.60 \% \\
2 . & 82.78 \% \\
\text { 3. } & 92.04 \% \\
\text { 4. } & 91.78 \% \\
\text { 5. } & 96.73 \%\end{array}$ \\
\hline
\end{tabular}

c) Gravanis et al. [37]: used the UNBiased dataset with 3,404 articles with 2,004 real news and 1,400 fake ones and achieved an accuracy up to $95 \%$ using the SVM classifier. They used 57 linguistic features with the Word2vec WE method. They also compared their method on other datasets (i.e., Kaggle-EXT, BuzzFeed, Politifact, McIntire) and achieved an accuracy of up to $99.0 \%, 72.70 \%, 84.7 \%$, and $81 \%$ respectively. They claimed of using a biased KaggleEXT dataset (in year 2018) with real news from one source only, which produced a constant performance regardless the number of features (i.e., up to $99 \%$ accuracy even by using a single linguistic feature, such as typo).

d) WELFake: applied on a larger dataset with over 72,000 news achieved a higher accuracy of $96.73 \%$ compared to the related methods. For a fair comparison, we separately applied WELFake model on the four smaller datasets (i.e., Kaggle, McIntire, Reuters, Buzzfeed) too. Compared to [37] (the better proven method among [48], [49], [37]) on the Buzzfeed and McIntire datasets, WELFake improved the accuracy from $72.7 \%$ to $82.7 \%$, respectively from $81 \%$ to $91.78 \%$.

\section{CONCLUSIONS AND FUTURE WORK}

We presented a new model called WELFake for text fake news detection. For this purpose, we prepared a larger dataset called WELFake with over 72,000 news articles combining four open source datasets (i.e., Kaggle, McIntire, Reuters, BuzzFeed) to reduce their individual limitation and bias. 
Afterwards, we analyzed over 80 linguistic features from stateof-the-art works and selected twenty significant ones to minimize the computational complexity and increase the standard classifiers' accuracy. We applied two WE-based methods (i.e., TF-IDF, CV) over these linguistic features using six ML models (i.e., KNN, SVM, NB, DT, Bagging, AdaBoost) and found out that CV produces a better overall accuracy than TFIDF with a SVM model. We therefore used CV over LFS and classified the twenty features based on four categories: writing pattern, readability index, psycho-linguistics, and quantity.

As the number of predictors that participate in voting classifier needs to be odd, we prepared three LFS by distributing the twenty selected features in a balanced manner across these categories. Afterwards, we embedded CV with these LFS and applied all six ML models. We determined the most accurate ML model and took its predicted results from each WE-enabled LFS dataset for voting classification. We finally applied the result of this voting classifier to the next level voting classification with best model results of TF-IDF and CV over LFS and obtained the final classification. Experimental results show that the WELFake model produces a high $96.73 \%$ accuracy on the WELFake dataset. To further analyze its advantage we compared it with two state-of-the-art works and found out that it improves the overall accuracy by $1.31 \%$ compared to BERT and $4.25 \%$ compared to CNN models. The proposed WELFake model also improved the accuracy by up to $10 \%$ on the McIntire and BuzzFeed datasets [37]. We also analysed the performance of different ML models in terms of accuracy, precision, recall and F1-score, and found out that SVM produced most accurate results. Finally, our frequency-based model focused on analyzing writing patterns outperformed predictive-based related works implemented using the Word2vec WE method by up to $1.73 \%$.

We plan to extend our work in the future with other factors like knowledge graphs and user credibility for further verification of the output generated by the WELFake model.

\section{ACKNOWLEDGMENT}

The ARTICONF project funded by the European Union's Horizon 2020 research and innovation program under the grant agreement number 825134 partially supported this work.

\section{REFERENCES}

[1] Jiang, J. Wu, F. Li, G. Wang, and H. Zheng, "Trust evaluation in online social networks using generalized network flow," IEEE Transactions on Computers, vol. 65, no. 3, pp. 952-963, Mar. 2016.

[2] Alrubaian, M. Al-Qurishi, A. Alamri, M. Al-Rakhami, M. Hassan, and G. Fortino, "Credibility in online social networks: A survey," IEEE Access, vol. 7, pp. 2828-2855, Dec. 2018.

[3] S. Ranganath, S. Wang, X. Hu, J. Tang, and H. Liu, "Facilitating time critical information seeking in social media," IEEE Transactions on Knowledge and Data Engineering, vol. 29, no. 10, pp. 2197-2209, Oct. 2017.

[4] Zhang, R. Sun, X. Wang, and C. Zhao, "A situational analytic method for user behavior pattern in multimedia social networks," IEEE Transactions on Big Data, vol. 5, no. 4, pp. 520-528, Dec. 2019.

[5] M. Schudson and B. Zelizer, "Fake news in context," in Understanding and Addressing the Disinformation Ecosystem, Pennsylvania: Annenberg School for Communication, Apr. 2017, pp. 1-4.

[6] S. Zaryan, "Truth and trust : How audiences are making sense of fake news," in Media and Communication Studies, Masters Dissertation, Sweden, Jun. 2017.
[7] S. Vosoughi, D. Roy, and S. Aral, "The spread of true and false news online," Science, vol. 359, no. 6380, pp. 1146-1151, Mar. 2018.

[8] R. Sequeira, A. Gayen, N. Ganguly, S. Dandapat, and J. Chandra, "A large-scale study of the twitter follower network to characterize the spread of prescription drug abuse tweets," IEEE Transactions on Computational Social Systems, vol. 6, no. 6, pp. 1232-1244, Dec. 2019.

[9] "Politifact news dataset," http://www.politifact.com/, online; accessed 31 March 2020.

[10] "The washingtonpost fact checker," https://www.washingtonpost.com/news/fact-checker, online; accessed 31 March 2020.

[11] "Fact check," https://www.factcheck.org/, online; accessed 31 March 2020.

[12] "Snopes," https://www.snopes.com/, online; accessed 31 March 2020.

[13] "Truthorfiction," https://www.truthorfiction.com/, online; accessed 31 March 2020.

[14] "Fullfact," https://fullfact.org/, online; accessed 31 March 2020.

[15] "Hoax slayer," http://hoax-slayer.com/, online; accessed 31 March 2020.

[16] "Viswas news," http://www.vishvasnews.com/, online; accessed 31 March 2020.

[17] "Factly," https://factly.in/, online; accessed 31 March 2020.

[18] L. Shi, L. Liu, Y. Wu, L. Jiang, M. Kazim, H. Ali, and J. Panneerselvam, "Human-centric cyber social computing model for hot-event detection and propagation," IEEE Transactions on Computational Social Systems, vol. 6, no. 5, pp. 1042-1050, Oct. 2019.

[19] M. Glenski, T. Weninger, and S. Volkova, "Propagation from deceptive news sources who shares, how much, how evenly, and how quickly?" IEEE Transactions on Computational Social Systems, vol. 5, no. 4, pp. 1071-1082, Dec. 2018.

[20] E. Lancaster, T. Chakraborty, and V. Subrahmanian, " $M A L T$ ? : Parallel prediction of malicious tweets," IEEE Transactions on Computational Social Systems, vol. 5, no. 4, pp. 1096-1108, Dec. 2018.

[21] P. K. Verma and P. Agrawal, "Study and detection of fake news: $P^{2} C^{2}$ based machine learning approach," in International Conference on Data Management, Analytics and Innovation, vol. 1175. Springer Singapore, Sept. 2020, pp. 261-278.

[22] Z. Jin, J. Cao, Y. Zhang, J. Zhou, and Q. Tian, "Novel visual and statistical image features for microblogs news verification," IEEE Transactions on Multimedia, vol. 19, no. 3, pp. 598-608, Mar. 2017.

[23] B. Ratner, "The correlation coefficient: Its values range between $+1 /-1$, or do they?" Journal of Targeting, Measurement and Analysis for Marketing, vol. 17, no. 2, pp. 139-142, Jun. 2009.

[24] A. De Salve, P. Mori, B. Guidi, and L. Ricci, "An analysis of the internal organization of facebook groups," IEEE Transactions on Computational Social Systems, vol. 6, no. 6, pp. 1245-1256, Dec. 2019.

[25] P. K. Verma, P. Agrawal, and R. Prodan, "WELFake dataset for fake news detection in text data," 2021, (Version: 0.1) [Data set], Zenodo.

[26] V. Madaan and A. Goyal, "Predicting ayurveda-based constituent balancing in human body using machine learning methods," IEEE Access, vol. 8, pp. 65060-65070, Apr. 2020.

[27] G. Mingchen Li, Y. Clinton, and Miao, "Short text classification via knowledge powered attention with similarity matrix based CNN," Feb. 2020, pp. 1-10. [Online]. Available: https://arxiv.org/abs/2002.03350v2

[28] C. Sun, X. Qiu, Y. Xu, and X. Huang, "How to fine-tune bert for text classification?" in Chinese Computational Linguistics, vol. 11856, Feb. 2020, pp. 194-206. [Online]. Available: https://arxiv.org/abs/1905.05583

[29] K. Dzmitry Bahdanau, Y. Cho, and Bengio, "Neural machine translation by jointly learning to align and translate," in $3^{\text {rd }}$ International Conference on Learning Representations, 2015, pp. 1-15. [Online]. Available: https://arxiv.org/abs/1409.0473

[30] Y. Chen, N. Conroy, and V. Rubin, "Misleading online content: Recognizing clickbait as false news," in ACM on Workshop on Multimodal Deception Detection (WMDD'15). New York, USA: Association for Computing Machinery, Nov. 2015, pp. 15-19.

[31] P. Bourgonje, J. Schneider, and G. Rehm, "From clickbait to fake news detection: An approach based on detecting the stance of headlines to articles," in EMNLP Workshop: Natural Language Processing meets Journalism. Copenhagen, Denmark: Association for Computational Linguistics, Sep. 2017, pp. 84-89.

[32] H. Rashkin, E. Choi, J. Jang, S. Volkova, and Y. Choi, "Truth of varying shades: Analyzing language in fake news and political fact-checking," in Conference on Empirical Methods in Natural Language Processing. Copenhagen, Denmark: Association for Computational Linguistics, Sep. 2017, pp. 2931-2937.

[33] M. Alrubaian, M. Al-Qurishi, M. Hassan, and A. Alamri, "A credibility analysis system for assessing information on twitter," IEEE Transactions 
on Dependable and Secure Computing, vol. 15, no. 4, pp. 661-674, Aug. 2018.

[34] C. Castillo, M. Mendoza, and B. Poblete, "Information credibility on twitter," in $20^{\text {th }}$ International Conference on World Wide Web. New York, USA: Association for Computing Machinery, Mar. 2011, pp. 675684.

[35] D. Benjamin, D. Horne, and S. Adali, "This just in: Fake news packs a lot in title, uses simpler, repetitive content in text body, more similar to satire than real news," in $2^{\text {nd }}$ International Workshop on News and Public Opinion, Mar. 2017, pp. 1-9.

[36] R. K. Kaliyar, A. Goswami, and S. Narang, P an Sinha, "FNDNet - a deep convolutional neural network for fake news detection," Cognitive Systems Research, vol. 61, pp. 32-44, Jun. 2020.

[37] G. Gravanis, A. Vakali, K. Diamantaras, and P. Karadais, "Behind the cues: A benchmarking study for fake news detection," Expert Systems with Applications, vol. 128, pp. 201-213, Aug. 2019.

[38] K. Shu, L. Cui, S. Wang, D. Lee, and H. Liu, "defend: Explainable fake news detection," in KDD'19: Proceedings of the $25^{\text {th }}$ ACM SIGKDD International Conference on Knowledge Discovery \& Data Mining. New York, United States: Association for Computing Machinery, Jul. 2019, pp. 395-405

[39] R. Zellers, A. Holtzman, H. Rashkin, Y. Bisk, A. Farhadi, F. Roesner, and Y. Choi, "Defending against neural fake news," May 2019, pp. 1-21. [Online]. Available: https://arxiv.org/abs/1905.12616

[40] J. K. Burgoon, J. Blair, T. Qin, and J. Nunamaker, "Detecting deception through linguistic analysis," in $1^{\text {st }}$ NSF / NIJ Conference on Intelligence and Security Informatics. Berlin Heidelberg; Berlin, Heidelberg: Springer, May 2003, pp. 91-101.

[41] M. D. Vicario, W. Quattrociocchi, A. Scala, and F. Zollo, "Polarization and fake news: Early warning of potential misinformation targets," $A C M$ Transaction Web, vol. 13, no. 2, pp. 1-22, Apr. 2019.

[42] . Pérez-Rosas, B. Kleinberg, A. Lefevre, and R. Mihalcea, "Automatic detection of fake news,", in $27^{\text {th }}$ International Conference on Computational Linguistics. Santa Fe, New Mexico, USA: Association for Computational Linguistics, Aug. 2018, pp. 3391-3401.

[43] C. Buntain and J. Golbeck, "Automatically identifying fake news in popular twitter threads," in IEEE International Conference on Smart Cloud (SmartCloud'17), New York, USA, Nov. 2017, pp. 208-215.

[44] T. Mitra and E. Gilbert, "Credbank: A large-scale social media corpus with associated credibility annotations," in International AAAI Conference on Web and Social Media, Aug. 2015, pp. 258-267.

[45] A. Zubiaga, G. Wong Sak, M. Hoi, R. Liakataand, and Procter, "PHEME dataset of rumours and non-rumours," Oct. 2016.

[46] M. L. Newman, J. Pennebaker, D. Berry, and J. Richards, "Lying words: Predicting deception from linguistic styles," Personality and Social Psychology Bulletin, vol. 29, no. 5, pp. 665-675, May 2003.

[47] L. Zhou, J. Burgoon, J. Nunamaker, and D. Twitchell, "Automating linguistics-based cues for detecting deception in text-based asynchronous computer-mediated communications," Group Decision and Negotiation, vol. 13, no. 1, pp. 81-106, Jan. 2004.

[48] H. Ahmed, I. Traore, and S. Saad, "Detection of online fake news using n-gram analysis and machine learning techniques," in Intelligent, Secure, and Dependable Systems in Distributed and Cloud Environments, vol. 10618. Cham: Springer, Oct. 2017, pp. 127-138.

[49] K. Shu, S. Wang, and H. Liu, "Exploiting tri-relationship for fake news detection," Dec. 2018, pp. 1-10. [Online]. Available: https://arxiv.org/abs/1712.07709v1

[50] C. Burfoot and T. Baldwin, "Automatic satire detection: Are you having a laugh?" in ACL-IJCNLP 2009 Conference Short Papers. Suntec, Singapore: Association for Computational Linguistics, Aug. 2009, pp. $161-164$.

[51] B. Riedel, I. Augenstein, G. Spithourakis, and S. Riedel, "A simple but tough-to-beat baseline for the fake news challenge stance detection task," Apr. 2017, pp. 1-6. [Online]. Available: https://arxiv.org/abs/1707.03264

[52] W. Y. Wang, "Liar, liar pants on fire: A new benchmark dataset for fake news detection," in 55 ${ }^{\text {th }}$ Annual Meeting of the Association for Computational Linguistics, vol. 2. ACM, Aug. 2017, pp. 422-426.

[53] "Mcintire fake news dataset," https://github.com/lutzhamel/fake-news, online; accessed 15 April 2020.

[54] "Fake news kaggle dataset," https://www.kaggle.com/c/fakenews/data?select=train.csv, online; accessed 15 April 2020.

[55] "Benjamin political news dataset," https://github.com/rpitrust/fakenewsdata1, online; accessed 15 May 2020.

[56] "Burfoot satire news dataset," http://www.csse.unimelb.edu.au/research/lt/ resources/satire, online; accessed 15 May 2020.

[57] "Buzzfeed news dataset," https://github.com/BuzzFeedNews/2016-10facebook-fact-check/tree/master/data, online; accessed 15 May 2020.
[58] "Credbank dataset," http://compsocial.github.io/CREDBANK-data, online; accessed 15 May 2020

[59] "Fake news challenge dataset," https://github.com/FakeNewsChallenge/fnc1, online; accessed 15 May 2020.

[60] "Fakenewsnet dataset," https://github.com/KaiDMML/FakeNewsNet, online; accessed 15 May 2020.

[61] "Liar dataset," https://www.cs.ucsb.edu/ william/data/liar_dataset.zip, online; accessed 15 May 2020.

[62] T. M. Mitchell, Machine Learning. New York: McGraw-Hill, 1997.

[63] G. Shmueli, N. R. Patel, and Bruce, Data Mining for Business Intelligence: Concepts, Techniques, and Applications in Microsoft Office Excel with XLMiner. Wiley, 2007.

[64] "Introduction to tf-idf," http://www.tfidf.com/, online; accessed 15 June 2020.

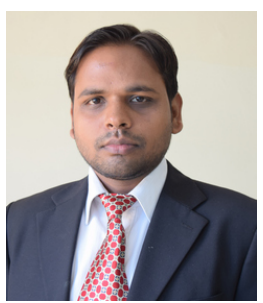

Pawan Kumar Verma received his M.Tech in Computer Science in 2010 from Jaypee University of Information Technology, India. He has more than eight years of teaching and research experience in opinion mining, pattern recognition, image processing and artificial intelligence. He is currently a Ph.D. scholar at the Lovely Professional University, India. $\mathrm{He}$ has Author/Co-Author 8+ research papers in various international conferences and journals of repute. He is a member of IEEE.

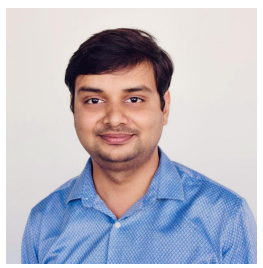

Prateek Agrawal received his $\mathrm{PhD}$ from IKGPunjab Technical University, India. He is currently a postdoctoral researcher at the University of Klagenfurt, Austria and associate professor at the School of Computer Science Engineering, Lovely Professional University, India. He authored more than 60 research papers in various peer reviewed journals and conferences. His research areas are natural language processing, machine learning, image processing, scheduling and parallel processing.

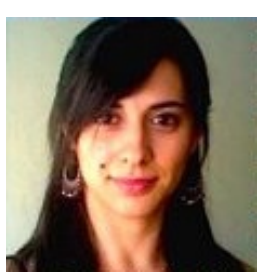

Ivone Amorim obtained her $\mathrm{PhD}$ degree in Computer Science in 2016 from the University of Porto, Portugal. Currently she is a researcher at MOG Technologies. She researched in the last years at several Portuguese institutions in the area of mathematics and its applications to several fields like robotics, power systems and cryptography. She participated in several national and international projects. She is an associated member of the CMUP mathematical research center at the University of Porto, Portugal.

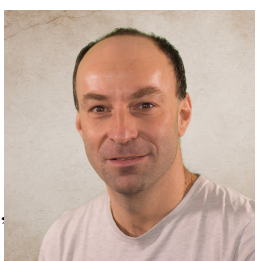

Radu Prodan received the $\mathrm{PhD}$ degree from the Vienna University of Technology in 2004. He was a associate professor until 2018 at the University of Innsbruck, Austria. He is currently professor in distributed systems at the Institute of Software Technology, University of Klagenfurt, Austria. He is the coordinator of the European Horizon 2020 project ARTICONF that researches a toolset for trustworthy, resilient and globally sustainable decentralised applications, with special focus on social media networks. He was involved in numerous national and European projects with a total budget of over $€ 6$ million. He authored more than 200 publications and received two IEEE best paper awards. 